\title{
Enquête
}

Archives de la revue Enquête

5 | 1997

Débats et controverses

\section{Margaret Mead et la sexualité à Samoa}

Du consensus anthropologique au débat ethnographique

Margaret Mead and Sexuality in Samoa. From Anthropological Consensus to

Ethnographical Debate

\section{Serge Tcherkézoff}

\section{OpenEdition \\ Journals}

Édition électronique

URL : https://journals.openedition.org/enquete/1203

DOI : 10.4000/enquete. 1203

ISSN : 1953-809X

Éditeur :

Cercom, Éditions Parenthèses

Édition imprimée

Date de publication : 1 septembre 1997

Pagination : 141-160

Référence électronique

Serge Tcherkézoff, « Margaret Mead et la sexualité à Samoa », Enquête [En ligne], 5 | 1997, mis en ligne le 15 juillet 2013, consulté le 21 septembre 2021. URL : http://journals.openedition.org/enquete/1203 ; DOI : https://doi.org/10.4000/enquete.1203

Ce document a été généré automatiquement le 21 septembre 2021. 


\title{
Margaret Mead et la sexualité à Samoa
}

\author{
Du consensus anthropologique au débat ethnographique \\ Margaret Mead and Sexuality in Samoa. From Anthropological Consensus to \\ Ethnographical Debate
}

Serge Tcherkézoff

1 Depuis maintenant une quinzaine d'années, le monde anglo-américain des sciences sociales est secoué sporadiquement par une salve de tirs qui, à travers plusieurs dizaines d'analyses, de comptes rendus critiques et de réponses enflammées, visent Derek Freeman, professeur australien âgé aujourd'hui de presque soixante-dix ans et auteur en 1983 d'un livre $^{1}$ dont chaque chapitre aboutit à une conclusion identique : le premier livre de Margaret Mead, publié en 1928 et décrivant l'Adolescence à Samoa (Polynésie), livre qui rendit immédiatement célèbre une jeune étudiante de vingt-cinq ans, serait une suite éhontée de mensonges ou, au moins, d'erreurs flagrantes ${ }^{2}$. Dès sa parution, le livre de Freeman fut déclaré " unscientific » en assemblée générale de la toute puissante Association américaine d'anthropologie, ce qui ne s'était jamais vu auparavant. Si le point de vue de Freeman n'est défendu que par une petite minorité mais parfois avec de puissants moyens, tel un film ou une pièce de théâtre à succès, montée en 1996 dans la salle principale de Sydney -, le débat a déjà suscité la publication de cinq livres, pour réunir articles et prises de positions, ou pour évaluer le débat lui-même. Il est désormais nécessaire d'en mener l'analyse; son abord peut suivre des voies différentes.

2 Il serait possible de chercher à comprendre sa violence, sa durée, l'ampleur des engagements suscités. Une autre étude pourrait reprendre l'analyse des débuts mêmes de M. Mead dans l'anthropologie; les informations sont désormais abondantes, recueillies par Freeman, ou offertes par les nombreuses biographies de Mead. Quelques cours de psychologie, la rencontre avec Ruth Benedict, deux ans d'anthropologie (1923-1925), une thèse de 90 pages sur la culture matérielle en Polynésie, à partir de livres déjà publiés, et portant aux neuf dixièmes sur la Polynésie orientale, sous la 
direction de Franz Boas; puis un contrat de recherche d'un an sur le problème des déterminations du comportement adolescent, le choix de la Polynésie malgré les réticences de ses aînés, Benedict et surtout Boas, directeur du contrat de recherche, qui ne voulait pas que Mead aille si loin - mais celle-ci s'accrochait à une image façonnée à partir de ses lectures sur les Marquises et élaborait un projet d'enquête aux Tuamotu (Polynésie française), toujours donc en Polynésie orientale; enfin le fait de se retrouver à Samoa sans l'avoir voulu, parce que Boas, après avoir accepté à contre-cœur la Polynésie, exige pour la jeune Mead un «terrain » relativement facile d'accès et sous administration américaine.

3 Une autre approche consisterait à observer comment Freeman se fait le champion d'une investigation à la manière du logicien Karl Popper - auteur qu'il cite à longueur de pages -, comment il refuse de faire une monographie pour ne pas verser dans les "interprétations», comment il dit vouloir rester sur le terrain de la "vérité falsifiable » et choisit ainsi une démarche peu commune. En effet, des longues enquêtes inédites conduites à Samoa en 1940-1943 et 1965-1967, Freeman tire une série de cas histoires de vie, événements -, qui constituent autant de contre-exemples aux généralisations que Mead propose à la fin de chaque chapitre de Adolescence à Samoa, ainsi que dans l'introduction et la conclusion ${ }^{3}$. Le résultat est désastreux à double titre.

D'une part, le lecteur extérieur se trouve confronté à une alternative caricaturale lorsqu'il tente de se représenter la culture samoane: soit l'atmosphère d'« amour libre » et de " détachement » devant toute idée de règles sociales suggérée par Mead, soit une culture de la violence, de l'agression sexuelle, du viol, et de l'oppression par les chefs, à laquelle ses « contre-exemples » ont réduit la subtile et profonde connaissance de Freeman sur Samoa. Voilà le résultat d'une démarche strictement "poppérienne " dans nos disciplines.

D'autre part, Freeman, toujours au nom de la "vérité », suggère que l'erreur de Mead tient précisément à sa conception d'une discipline qui n'avancerait que par "interprétations», alors qu'il faudrait introduire dans l'analyse sociale les déterminations scientifiques du comportement humain, qui tiennent à la nature biologique de l'homme. Non seulement, le personnage le plus célèbre des sciences sociales américaines était attaqué, qui plus est après sa mort (Mead est décédée en 1978), mais encore toute la discipline fondée par Boas se trouvait soumise à la critique. Aussi, même ceux qui savaient ou se doutaient que Mead avait fortement simplifié les choses dans ses généralisations sur "Samoa » - un travail d'une étudiante totalement inexpérimentée et obéissant aux modèles affreusement simplistes de Benedict - ne purent que défendre le livre de Mead contre Freeman, fermant ainsi toute possibilité de discussion sur la culture samoane.

6 Pourtant, c'est bien là l'essentiel. Le lecteur pourra se reporter à d'autres publications à ce sujet ${ }^{4}$. Très grossièrement, on peut dire ceci. Après les changements issus de l'évangélisation (1830-1850), la culture samoane ne connaît pas de bouleversement jusqu'au début des années soixante ${ }^{5}$; elle a construit une distinction fondamentale entre deux conceptions des relations hommes-femmes, dont nous donnons ici les grandes lignes.

7 La première repose sur l'univers de la « lumière ", domaine de la maison et du village, conformément à la grande opposition cosmogonique polynésienne entre la Nuit et le Jour. À Samoa, les maisons, sans murs, sont d'abord des lieux publics, des salles de réunion dans lesquelles on peut accessoirement dormir. L'humanité y est répartie en 
«familles» se reproduisant par la conservation cultuelle d'un stock de noms fondateurs. Ces noms et leur conservation reposent sur la perpétuation de rapports «frère-sœur ». Au bout d'une génération, les liens d'affinité sont intégrés dans cette consanguinité très particulière : le mari est assimilé aux frères de sa femme, la femme aux frères (et non aux sœurs) de son mari. Si le mariage est symboliquement ignoré, la sexualité l'est encore plus. Il est contre la coutume de faire l'amour dans les maisons, et même de parler de sexualité devant des tiers (même dans le couple légalement marié), car tout le monde est assimilé, pour dire bref, à des frères et sœurs. Par ailleurs, pour des raisons tenant aux conceptions anciennes de la reproduction, les femmes doivent arriver vierges au mariage, condition pour produire des « enfants sacrés » (appellation par le frère des enfants de sa sœur). Mead n'a rien vu de tout cela: elle n'a jamais participé longuement à la vie quotidienne et cérémonielle d'un village, car elle habitait au poste américain de l'île et conduisait des entretiens, par interprètes, avec une cinquantaine de jeunes filles.

8 La seconde concerne le monde de l'« obscurité », temps de la nuit, mais aussi n'importe quel moment quand on est "caché ». Ce monde rejoint le monde cosmologique primordial, celui de la «nature » avant que l'intervention de la «culture » n'établisse des « cercles de lumière " : ces cercles sont les maisons et les villages. Dans ce monde, l'humain et l'animal ne se distinguent pas. Tout est sexué-et-sexuel, il n'y a que du "mâle-et-femelle ", et la distinction de genre y est conçue uniquement en référence au rapport sexuel (l'acte sexuel). Un des résultats surprenants mais fort logiques est une atmosphère de «liberté » sexuelle, du fait que les individus sont alors placés hors du social (au sens des cercles de lumière). Or, la hiérarchie, donc les interdits, n'ont de sens à Samoa que dans les cercles de lumière. À l'extérieur, il n'y a plus que des individus qui sont d'autant plus « libres » qu'ils sont « solitaires » et " cachés ».

9 À tout cela s'ajoute l'asymétrie des sexes, fondamentale, qui exprime à elle seule toute la domination masculine. Le vocabulaire et l'ensemble des attitudes, chez les deux sexes, distinguent farouchement la femme en tant que sœur, dont la sexualité doit être absente si elle n'est pas mariée (ou doit être passée sous silence si la femme est mariée, du moins quand cette femme séjourne dans son propre village), et la femme en tant que « femelle », à la fois réputée ayant " perdu » sa virginité (ce qui s'appelle une " chute ») et n'ayant comme identification que le pôle féminin du rapport défini par la représentation de l'acte hétérosexuel. L'homme au contraire n'est pas soumis à cette dichotomie inéluctable.

Le résultat est qu'il y a évidemment diverses aventures sexuelles adolescentes prémaritales, que les hommes n'y trouvent que plaisir et affirmation de soi, alors que les femmes sont constamment confrontées à un choix : l'appel de la «liberté » hors du monde ou la loi des rapports sociaux orientés par l'idéologie frère-sœur. Une des conséquences en est un imaginaire sexuel des jeunes filles, qui multiplie des aventures rocambolesques dans une brousse peuplée d'esprits, ce qui correspond très peu à la pratique réelle. Mead, tout à fait inconsciente de cette vaste dichotomie, a recueilli de nombreux récits (imaginaires) de jeunes filles et y a vu, bien à tort, le signe de valeurs sociales de liberté sexuelle. Au contraire, cette "liberté » n'a de sens que hors du social : dans des rencontres certes joyeuses mais qui ne peuvent se passer qu'en brousse et qui, surtout, quand elles aboutissent à une grossesse, se transforment souvent en drames : la jeune femme est morte de «honte » et craint la violence de son père et de ses frères. 
11 Dans ce cadre, nous nous limiterons ici à un aspect essentiel de ce débat, qui concerne fortement l'histoire de notre discipline. Comment un manuscrit outrageusement simpliste, rapporté par une jeune étudiante qui n'avait encore rien publié, est-il devenu le plus grand best-seller de tous les livres d'anthropologie, réimprimé sans interruption depuis soixante ans, dans seize pays et à plusieurs millions d'exemplaires? L'attaque portée par Freeman touche ainsi au cœur un consensus scientifique sans véritable faille.

\section{L'ethos samoan, à coup de généralités}

12 Le livre que Margaret Mead publie en 1928 entend décrire le «tempérament culturel » de Samoa, c'est-à-dire les tendances dominantes, les lignes de force de l'ethos propre à cette culture, en particulier tel qu'il se donne à voir chez les adolescents. L'analyse porte sur un groupe d'une cinquantaine de jeunes filles, dont l'auteur décrit le caractère, la biographie, les désirs et la vie sexuelle (supposée). Dans le détail, bien des éléments se laissent reconnaître comme "vraisemblables" pour qui a vécu à Samoa. Sans aucun doute, Mead a bien été dans ce pays. Si l'auteur sait varier ses observations, et donne à un certain nombre de jeunes filles une réelle individualité, sous sa plume, les généralisations, tirées de ces cas individuels, afin de définir l'ethos samoan, proposent un véritable cliché : sur ces îles, tout ne serait que paix, calme et volupté... Ce pays est le jardin d'Éden avant la Chute. Tous les habitants font de l'acte d'amour leur "passetemps favori », prenant juste le soin de placer un buisson ou un palmier devant eux pour ne pas trop s'exposer aux regards. En 1928, toute l'Amérique ou presque prit ce décor imaginaire pour une photographie réaliste. Or ce décor est lié à la fois à la formation initiale de Mead, et au cadre institutionnel de sa recherche.

Mead est persuadée qu'il y a des sociétés plus « simples » que d'autres parce que plus « primitives ». Elle est aussi une psychologue en fin d'études qui vient à l'anthropologie et considère que toute culture se construit autour de quelques choix d'ordre psychologique. La culture privilégie inconsciemment une tendance plutôt qu'une autre. L'anthropologue doit identifier cette "tendance dominante ». Il suffit de rappeler un passage célèbre, celui qui ouvre l'étude de Mead sur les trois cultures de NouvelleGuinée, publiée en 1935, à partir d'enquêtes qui commencent en 1928, dès que Coming of Age in Samoa est publié :

On ne peut manquer d'admirer, lorsqu'on étudie les sociétés primitives, l'extrême diversité des démarches de l'imagination humaine qui, s'emparant d'un nombre limité de données essentielles, a su construire ces magnifiques édifices que nous nommons civilisations. [...] Le milieu naturel [...] impose à l'homme le spectacle de ses contrastes [...] : le jour et la nuit, le cycle des saisons [...]. Son propre corps lui parle d'âge et de sexe, de consanguinité [...]. Il voit les animaux différents les uns des autres, de même les individus : le féroce et le tendre [...]. Chaque peuple ourdit cette trame de façon différente, comme s'il choisissait certains fils à l'exclusion des autres, et ne met en relief qu'un aspect des virtualités humaines. Ainsi, chez les uns, tout s'organise autour du moi vulnérable, prompt à saisir l'insulte ou à périr de honte. Pour les autres, c'est le courage inflexible, tels les Cheyennes qui ont inventé pour les craintifs une position sociale de statut particulièrement complexe, à seule fin de ne pas admettre qu'il y ait des poltrons parmi eux. Chaque civilisation primitive et homogène ne peut donner carrière qu'à quelques-unes des capacités de l'homme. Elle interdit ou pénalise toutes celles qui sont trop opposées ou trop étrangères à son orientation principale. [...] Chaque nouvelle génération se trouve 
façonnée, fermement et définitivement, selon la tendance dominante. [...] Voilà donc ce que j'ai voulu étudier chez les doux montagnards Arapesh, les féroces cannibales Mundugumor, les gracieux chasseurs de têtes Tchambuli ${ }^{6}$. Mead y découvre, dit-elle, une « civilisation simple et uniforme », dont la tendance est le «détachement" vis-à-vis de tout enjeu et une volonté constante "d'éviter les conflits ». Une des conséquences en est que, dans sa description, « aucun dilemme de cette sorte ne confronte la jeune Samoane. L'activité sexuelle est chose naturelle et agréable. On peut s'y adonner librement, dans les seules limites qu'impose le rang social : les filles de chefs et les épouses de chefs ne doivent se permettre aucun écart en dehors du mariage ${ }^{7}$ ». Mais, ajoute aussitôt Mead, ces filles de chef ne sont que quelques exceptions qui ne concernent pas «la classe populaire ». En règle générale, aucun conflit ne vient donc perturber les adolescents, et leur vie sexuelle en est le signe le plus évident. Mead en déduit la réponse à la question que son contrat lui demande d'étudier et y trouve la preuve de la justesse des positions culturalistes : la raison de cette adolescence facile ne peut tenir qu'à la tendance générale de cette culture du "détachement». Voilà un exemple net de la détermination culturelle opérant sur l'adolescence.

17 Mead rapporte ainsi deux messages, exprimés dans ses généralisations. Le premier est intentionnel et s'adresse aux scientifiques, en premier lieu à ceux qui l'ont envoyée dans le Pacifique, Boas et les siens : dans un cas au moins, la période de la vie qui paraît le mieux s'expliquer par les contraintes naturelles du développement physique dépend elle aussi de l'environnement culturel. Le second message s'adresse au grand public et concerne l'éducation des adolescents : pour faire le bonheur d'une communauté et de ses adolescents, il faut laisser parler la nature ; « l'activité sexuelle est chose naturelle et agréable ».

Dans l'Amérique des années vingt, qui commençait çà et là à soulever le joug du puritanisme, un tel message présenté comme un résultat scientifique est reçu avec reconnaissance et enthousiasme. Ce public ne retient guère le thème du débat entre partisans de la détermination culturelle ou naturelle; d'autant plus que, très habilement ou très naïvement, Mead dit bien que le choix culturel des Samoans de privilégier cette "chose naturelle » qu'est "l'activité sexuelle » rejoint au fond la nature. Il ne s'interroge pas sur une anthropologie des «tendances dominantes » dans 
les cultures. Il est simplement heureux de trouver un livre scientifique, préfacé par le grand universitaire Franz Boas, qui donne consistance au mythe déjà installé par d'autres écrits du siècle précédent : en Polynésie, tout n'est que paix, calme et volupté, dans les relations sociales et... sexuelles. Le changement de sous-titre exprime ainsi la réception américaine de l'ouvrage : A Psychological Study of Primitive Youth for Western Civilization devient rapidement A Study of Adolescence and Sex in Primitive Societies; les études que Mead publie en 1935 sur la Nouvelle-Guinée s'intituleront Sex and Temperament in Three Primitive Societies. Mead est désormais une spécialiste de la construction culturelle de la sexualité, et des enseignements que l'on peut en tirer pour l'éducation en Amérique.

\section{«Une journée à Samoa » : les modes de la généralisation}

19 Adolescence à Samoa commence par un chapitre destiné à informer, "Une journée à Samoa ». Classiquement, l'auteur décrit, par quelques exemples bien choisis, les événements ordinaires, qui illustrent les valeurs essentielles des habitants. L'exercice, périlleux, ne délivre qu'une généralité vue de loin ou de haut. Le récit fait trois pages, mais n'évoque pas moins de sept fois l'acte sexuel. S'agirait-il de la valeur culturelle dominante et du passe-temps social de base ? C'est le seul message que le lecteur peut retenir. S'il avait un doute, la couverture du livre (dans la première édition) le confirme dans cette idée : le soleil se couche, une femme entraîne un homme par la main vers des palmiers, les longs cheveux déroulés, la fleur de l'hibiscus accrochée dans l'épaisseur de sa coiffure ${ }^{8}$. Mead nous emmène donc pour une « journée » à Samoa.

Le matin

Le village s'éveille. [...] De l'abri des pirogues échouées, ou sous les palmiers, les amoureux se séparent et rejoignent furtivement leurs cases respectives. [...] Le village tout entier s'arrache au sommeil [...] et s'achemine vers la plage. [...] Des filles s'arrêtent, et l'on entend de petits rires : un propre à rien s'est dérobé cette nuit à la poursuite d'un père outragé, et, sûrement, la fille en sait plus long làdessus qu'elle ne le dit. Un garçon reçoit les moqueries d'un autre, qui lui a succédé dans les faveurs d'une fille.

L'après-midi

Les pêcheurs tirent leur pirogue au sec. [...] À regret les jeunes pêcheurs mettent de côté le "poisson tabou " qui doit être envoyé au chef. Mais, fièrement, ils garnissent de petits paniers en feuille de cocotier pour aller les offrir à leur belle.

Le soir

La moitié du village ira peut-être pêcher à la torche, et le récif [...] retentira des cris de triomphe et de déception, de taquineries, ou des protestations étouffées de pudeurs outragées. [...] Un groupe de jeunes gens dansera en l'honneur de quelque fille en visite. [...] De temps à autre, un couple se détache pour aller se perdre sous les arbres. [...] Enfin il ne restera plus que le doux grondement du récif et le murmure des amants, tandis que tout reposera jusqu'à l'aube'.

Dans ce récit, aucun autre thème concernant la vie quotidienne n'est ainsi répété sept fois de suite pendant ces quatre pages. Cette image de l'amour libre sous les palmiers est même la seule notation qui est reprise au cours des divers moments de la journée. D'ailleurs, un an plus tard, Mead écrit que, pour les Samoans, faire l'amour est «the pastime par excellence ${ }^{10} »$. L'intention de l'auteur est donc claire sur la leçon que le lecteur doit retenir, concernant la tendance dominante de la culture samoane : les actes liés aux obligations sociales sont accomplis «à regret » (préparer le poisson du chef), 
alors qu'on s'empresse de faire l'essentiel, à savoir les cadeaux entre amants. Il peut imaginer cette atmosphère où, certes, les amants se cachent "sous les palmiers » et rejoignent leurs cases « furtivement ", mais où les conversations sur les aventures de la nuit précédente envahissent néanmoins l'espace public, créant la même harmonie que celle entretenue pendant la nuit, entre le bruit des vagues sur les rochers et le murmure des amants enlacés.

Dans le reste du livre, Mead ne fait pas autrement. Régulièrement, des vignettes occupent une place cruciale, comme celle du jeune homme criant à sa "belle ", devant tout le monde, qu'il viendra la voir le soir dans son lit. Régulièrement aussi, Mead ponctue ses descriptions de généralisations : les Samoans ont élevé l'amour libre au rang d'un art - mais c'est le seul domaine artistique que leur culture reconnaisse -, l'idéal quotidien des jeunes filles est de fréquenter le plus d'amants possibles, etc.

Sur sa méthode générale, Mead est explicite dans l'introduction à son livre :

L'anthropologue se tourne vers les peuples primitifs, dont la société n'a jamais atteint la complexité de la nôtre : Esquimaux, Australiens, Océaniens, Pueblos. Il sait que plus une culture est simple, plus son analyse est aisée.

[Ainsi,] un spécialiste expérimenté peut comprendre la structure fondamentale d'une société primitive en quelques mois. [...]

M'attachant particulièrement aux fillettes et jeunes filles [...] pendant les neuf mois de mon séjour à Samoa, j'ai rassemblé de nombreuses données concernant la position sociale et la richesse des parents de ces filles, le nombre de leurs frères et de leurs sœurs, l'expérience sexuelle qu'elles avaient pu avoir [...].

Ces notions, peu mesurables, se sont révélées si peu variables d'un cas à l'autre, la vie de la jeune Samoane ressemble tellement à celle de sa voisine dans cette civilisation simple et uniforme, que je n'aperçois aucun inconvénient à généraliser, bien que mon enquête ait porté seulement, en fait, sur cinquante fillettes et jeunes filles des trois villages. [...]

J'ai passé sous silence les questions d'organisation politique qui n'intéressent pas la jeune fille et n'ont aucune influence sur elle ${ }^{11}$.

La dernière remarque permet à Mead de laisser de côté tous les interdits sociaux, comme ceux exigeant la virginité de la fiancée et, au XIX $x^{e}$ siècle, la cérémonie de la défloration, sous le prétexte que tout ceci est réservé aux filles de chef. Ce domaine réservé est une "organisation politique qui n'intéresse pas la jeune fille», la jeune fille prise comme «type idéal » au sens vague. Or, à Samoa, il n'y a aucune jeune fille qui ne soit pas « une fille de chef », car la notion de "chef » est simplement celle de chef de « famille étendue » (aiga), et tout adolescent appartient à une famille de ce genre.

Pour Mead, le rapport est évident entre, d'une part, une adolescence heureuse et, d'autre part, une société qui se définit à l'inverse de toutes les autres par le « détachement » vis-à-vis de toute idée de contrainte. Dans cette société de la liberté, la sexualité, « libre » elle aussi, permet d'éviter tous les ennuis psychologiques. On notera que Mead ne parvient à définir la culture samoane que par la négative (absence de contrainte, etc.). Elle avoue elle-même en être surprise : une société qui évite de faire ce que font toutes les autres est par définition bien rare. Mais ce caractère exceptionnel ne lui procure aucun doute sur sa méthode. Au contraire, elle n'hésite pas à l'affirmer :

Le champ laissé à l'expression sexuelle étant très large, il est difficile qu'apparaissent les complexes de culpabilité, qui sont, si fréquemment chez nous, causes d'inadaptation.

Ainsi l'amour sexuel est-il pour les Samoans un domaine familier, qui doit être traité comme un art et, par conséquent, exige une technique. De cette conception résultent des rapports personnels dont sont absentes névroses, frigidité et 
impuissance.

Si l'adolescence des Samoanes est si facile, si simple, c'est grâce à l'atmosphère de détachement, de désinvolture qui règne dans la communauté tout entière. Aux Samoa, personne ne joue gros, personne ne paie jamais très cher, personne n'est prêt à souffrir pour défendre ses convictions. [...] Par ce détachement, par cette façon d'éviter les conflits, les situations pénibles, les Samoa offrent un vif contraste non seulement avec l'Amérique, mais avec la plupart des civilisations primitives. Une société qui manque autant de profondeur ne peut produire ni de puissantes personnalités ni de grands artistes ; mais s'il est permis, à ce titre, de regretter une telle attitude, nous devons reconnaître néanmoins qu'elle contribue notablement à assurer une transition facile entre l'état de fillette et celui de femme ${ }^{12}$.

Dans les années cinquante et soixante, les intellectuels samoans qui lurent ces lignes, lorsque les premières bourses d'études supérieures leur furent attribuées par la Nouvelle-Zélande, furent consternés. Le monde occidental qui les invitait à des études universitaires leur expliquait que ce travail universitaire analysant le caractère superficiel de leur culture («ni puissantes personnalités ni grands artistes») était... d'une grande profondeur et constituait un modèle de démarche scientifique. Ils en ont gardé un profond ressentiment, d'autant plus grand que le résultat aboutit à faire de Samoa une exception mondiale, selon les mots de Mead elle-même ${ }^{13}$.

\section{La construction d'un consensus scientifique en anthropologie et ses rapports à l'exotisme occidental}

Il reste à comprendre pour quelle raison ce livre, au lieu d'être tourné en ridicule, fut consacré non seulement par le grand public, mais par la communauté scientifique. Deux circonstances expliquent ce phénomène : une image déjà bien dessinée chez les scientifiques occidentaux des caractères des cultures polynésiennes en général, un combat théorique important mené par Franz Boas, dont Mead fut le meilleur soldat.

La préface que Boas écrit pour coming of Age in Samoa a joué un rôle essentiel. Professeur prestigieux qui a installé l'anthropologie à l'Université Columbia (New York) en 1899, premier professionnel de l'enquête de terrain dans l'anthropologie américaine (chez les Esquimaux en 1884, chez les Indiens de Colombie britannique en 1886), Boas écrit en 1928 que chacun doit être reconnaissant à Margaret Mead pour avoir réussi à rapporter une description « aussi claire et lucide » de l'adolescence samoane, grâce à des investigations de terrain où elle est parvenue "à s'identifier de façon si complète avec la jeunesse samoane ", avant de conclure: "The results of her painstaking investigation confirm the suspicion long held by anthropologists, that much of what we ascribe to human nature is no more than a reaction to the restraints put upon by our civilization. » Boas intègre très vite dans ses propres livres les conclusions de Mead, comme dans son Anthropology and Modern Life (1928) qui eut un grand impact. À ce moment, Boas jouit d'une immense réputation de sérieux dans son usage de la méthode d'enquête ethnographique. S'il dit que l'enquête de Mead est "lucide", personne ne peut émettre un doute. Mais comment Boas a-t-il pu croire que les propos tenus par Mead révélaient une enquête «lucide »?

Dans l'Amérique de cette époque et, plus largement, en Occident, l'image de la Polynésie est celle d'un paradis sexuel de jeunes adolescentes. La Polynésie, c'est la « vahiné »! Le cliché durera encore au moins cinquante ans. En 1954, dans un livre qui 
se veut scientifique et qui rassemble les notations des voyageurs anciens et des observations personnelles sur de nombreux archipels de Polynésie orientale et centrale traitant des représentations et pratiques de «l'amour dans les mers du Sud », Bengt Danielsson écrit : «If, then, we find that the Polynesians were often astonishingly free in sexual matters, we can safely assume that it was not because rules of conduct were entirely lacking [...] the Polynesians were not moral anarchists, but radier slaves of custom. [...] As might be expected of a people so emphatic and appreciative an attitude towards sex life as the Polynesians, they made no attempt to suppress sexuality in the younger generation; parents, indeed, encouraged their children in free experimentalizing and realistic play. [...] It is not surprising in the circumstances that most of the well-known complexes and maladjustments from which our children suffer are still unknown in Polynesia ${ }^{14}$. » Bref, l'amour libre serait bien une valeur culturelle propre à la Polynésie et élaborée dans chacune des sociétés de cette civilisation.

Les récits $d u X^{x} I^{e}{ }^{e}$ et $d u x_{x}{ }^{e}$ siècle n'ont pas encore été soumis à la critique. Et, à l'époque de Mead, les livres scientifiques en langue anglaise, qui portent davantage sur les archipels orientaux (Marquises, etc.), insistent lourdement sur la promiscuité sexuelle. Les travaux sur les Marquises d'un auteur réputé des plus sérieux, E. S. Craighill Handy, du Bishop Museum d'Honolulu, sont alors considérés comme valides pour toute la Polynésie : « During this phase of their life youths and maids were totally free sexually. [...] It seems to be an irrésistible instinct of native of both sexes to run wild for a few years after adolescence, in pursuit of amusement in general, but of the satisfaction of their abundant sexual appetite in particular. A girl was looked down upon in native society if she did not run wild in this way [...]. A mother's pride was greatest and it was a matter of boasting if her daughter had the greatest number of suitors in her train ${ }^{15}$.»

Dans son livre sur la « religion polynésienne », Handy lui-même mêle les exemples de Samoa et des Marquises et se prête ainsi à la généralisation. Il raconte à la suite que, au festival tenu en l'honneur du dieu Fee, dans une région de Samoa, on observe des «boxing and wrestling matches, dances and obscenities which followed each other in rapid succession »; aux Marquises, il y avait aussi des "festivals held in honour of the spirits of the departed [... which] culminated in Lupercalian orgies in which the sexual instincts of all those taking part, strengthened by a long period of enforced continence preceding those rites, were released and given unrestrained and promiscuous freedom ${ }^{16}$ ».

Les faits samoans sont rapportés par Handy d'après les missionnaires qui, souvent, se fondent sur une rumeur, et sont amalgamés au thème très répandu à cette époque de la promiscuité sexuelle aux Marquises. Mead elle-même a subi directement l'influence de Handy, plus que les autres scientifiques de l'époque : ses livres furent l'une des sources principales de la préparation littéraire de Mead, et Handy en personne donna à Mead des cours de civilisation polynésienne, quand Mead s'arrêta à Honolulu en 1925, en route pour Samoa. Se trouvent ainsi abusivement mêlés des récits sur des rites appartenant à des confréries de types "Arioi, Kaioi " propres à la région orientale (Tahiti, Marquises, Tuamotu) et des notations de missionnaires sur Samoa, qui se réduisent à condamner des débordements qui ne sont jamais décrits. Mais Handy ne faisait pas le détail, ni dans ses livres, ni dans les conseils qu'il donne personnellement à Mead. Peu après, Ralph Linton, à propos de la confrérie marquisienne des Kaioi, composée de jeunes gens, écrit lui aussi que « the girls of the kaioi group were expected 
to entertain visiting males sexually, and they took great pride in the number of men they could satisfy in an evening. Except for taboos in the case of siblings and parents, there was complete sexual licence among these young people ${ }^{17}$ ».

Des descriptions comme celle d'une "journée à Samoa " semblaient alors normales, une fois précisé que "Samoa " c'est la "Polynésie». R. H. Lowie l'affirme dans son compte rendu de l'ouvrage: "Miss Mead's graphic picture of Polynesian free love is convincing. It falls into line with the reports of early travellers. [...] It is supported by Dr Handy's evidence from the Marquesas ${ }^{18}$. » Or R. H. Lowie est à ce moment une figure majeure : conservateur-adjoint pour l'anthropologie à l'American Museum of Natural History depuis 1917, il a travaillé avec Kroeber, lui-même l'un des tout premiers étudiants de Boas; il enseigne à Berkeley et dirige la première revue américaine d'anthropologie, American Anthropologist.

"Free love", le mot est lâché par les professionnels, et, parce qu'il s'agit de la "Polynésie ", il n'étonne personne; comme le dit Lowie, Mead ne fait que confirmer ce qu'on savait. Il n'arrêtera plus de résonner. Samoa devient un cas-type pour les ouvrages généraux. En 1962, la rumeur a déjà subi une profonde déformation. Dans un ouvrage général sur la culture, Understanding Culture, on trouve écrit que Samoa est une culture qui connaît la «institutionalized premarital sexuality ${ }^{19}$ ». Samoa devient un exemple privilégié pour les discussions radiophoniques. En 1962, à la BBC, on dit que, grâce à Mead, nous savons que "every young Samoan [...] has had many sexual expériences before marriage ${ }^{20} »$. Désormais, quand un Samoan est interviewé, on ne lui demande pas s'il est marié, mais d'emblée on s'enquiert du nombre d'amants/amantes qu'il/elle fréquente.

En 1928, Boas n'a donc aucune raison de croire que Mead invente ou s'est méprise et, dans ces conditions, il ne peut que se réjouir du résultat. S'il le fait savoir haut et fort en préfaçant le premier livre d'une toute jeune étudiante, c'est que Mead apporte la preuve tant attendue que l'adolescence peut être radicalement différente ailleurs. Il faut bien savoir à quel point le combat - c'est le mot juste - était âpre entre le clan des Boasiens et les partisans de l'explication des faits humains par la nature, y compris les faits que nous appelons aujourd'hui de société. Ces partisans de l'explication par la biologie et par l'évolution naturelle faisaient le lit des racistes et des eugénistes qui voulaient privilégier la race blanche et même l'améliorer par sélection. Boas et les siens quant à eux faisaient flèche de tout bois pour illustrer la thèse adverse dès qu'un exemple permettait de montrer l'influence de la détermination culturelle. Ce que rapportait Mead permettait de décocher des flèches plus solides que jamais. Boas fait donc une préface - ce qui ne lui est pas coutumier pour le premier travail d'une étudiante - et tout le clan utilise le livre comme un drapeau ${ }^{21}$.

R. Benedict, l'assistante de Boas qui a soutenu sa thèse avec lui en 1922, est la première enseignante de Mead et devient son amie intime. Elle fait plusieurs comptes rendus qui considèrent ces résultats, appuyés "sur des faits concrets ", "plus convaincants que n'importe quel argument a priori ${ }^{22} »$. Dès le début, la qualité de l'ethnographe est reconnue, au-delà même du cercle des amis. Malinowski, la référence en matière d'enquête ethnographique de longue durée, écrit dans The Nation, dès la sortie du livre, que le travail de Mead est « an absolutely first-rate pièce of descriptive ethnography ${ }^{23}$ ». Mead bénéficie d'un coup de chance: Malinowski s'apprêtait à publier sa propre étude sur la part culturelle de la sexualité en Mélanésie, et il trouvait dans le livre de Mead des conclusions qui s'alliaient objectivement aux siennes ${ }^{24}$. 
La réputation de Mead sort ainsi d'emblée du cercle boasien. Mead, désormais célèbre, publie deux ans plus tard, en 1930, une monographie sur l'organisation sociale de Samoa $^{28}$. Selon nos connaissances actuelles, le travail est mauvais, entaché de graves erreurs. Mais personne n'imagine à l'époque que cela puisse être le cas, et Mead devient aussi la référence sur la parenté polynésienne. En 1936, soit seulement six ans plus tard, il parait ainsi normal à R. Firth de comparer Mead à Malinowski et à Radcliffe-Brown. Pourtant, Firth est à l'écart du groupe américain. Né en Nouvelle-Zélande, il fait ses études en Angleterre; il publie un premier livre sur les Maori en 1929. Il conduit une longue enquête de terrain en 1928-1929 à Tikopia, minuscule île polynésienne en zone mélanésienne, qui offrait l'intérêt immense de n'avoir été contactée par les missionnaires que vingt ans plus tôt. Il publie en 1936 le chef-d'œuvre qu'est We The Tikopia. Il est donc tout à fait le contemporain de Mead. Or, dans l'introduction de sa monographie de 1936, quand Firth annonce qu'il va traiter de la parenté et qu'il cite les travaux de ceux qui «have done much to rescue the sociology of kinship from hypotheses of origin", en nommant naturellement ceux qui furent ses enseignants et dont la renommée est grande, comme Malinowski, il inclut le nom de Mead: "The work of Malinowski, Radcliffe-Brown, Margaret Mead, Elkin, Loyd Warner and Fortune has done much ${ }^{29}$. " Après avoir seulement publié deux ouvrages sur Samoa puis une étude sur les types psychologiques dans trois sociétés de Nouvelle-Guinée (en 1935), Mead est donc désormais classée non seulement parmi les grands mais encore parmi ceux qui ont grandement contribué à l'étude de l'organisation sociale, en particulier de la parenté, et cela même par les spécialistes britanniques. En 1950, Evans-Pritchard, un africaniste cette fois, porte le livre de Mead aux nues, dans ses conférences radiophoniques sur l'anthropologie sociale ${ }^{30}$. Dans ces mêmes années cinquante, la notoriété de Mead est telle que, lorsque l'Unesco cherche une personnalité pour coordonner un travail qui a trait aux modèles culturels dans le monde, même s'il s'agit de régions où Mead n'a jamais mis les pieds, c'est à elle qu'on pense immédiatement, afin d'avoir un livre "prepared by an outstanding group of international social scientists under the direction of the renowned anthropologist, Margaret Mead ${ }^{31}$ ».

Les choses ont donc été très vite. En 1928, Boas préface le travail de Mead et l'aide à trouver un éditeur commercial. Le livre connaît le succès. Quelques mois plus tard, 
Malinowski en fait un compte rendu laudateur. Sept ans plus tard, Mead est tout simplement comparée au même Malinowski. Ensuite, plus rien n'arrêtera cette renommée, qui franchit l'Atlantique, puis les limites du monde anglophone. La préface à l'édition italienne de Coming of Age in Samoa, écrite en 1964, nous dit que l'auteur de ce livre fait partie de « ces personnalités fameuses que Ellis, Malinowski, Russel, Boas, Brill n'ont pas hésité à désigner comme l'une des personnes les plus importantes de notre temps ». Le livre de Mead marque "une date décisive. C'est la première fois qu'une étude intensive et non extensive est menée dans une civilisation primitive [...] avec une participation intime à la vie du groupe ${ }^{32}$. »

40 Aucune critique ethnographique d'envergure n'a donc été formulée contre les écrits de Mead sur Samoa jusqu'en 1983, date de publication du livre de D. Freeman. On peut noter seulement quelques critiques partielles au début des années soixante, de la part de trois ou quatre chercheurs qui ont séjourné à Samoa (dont Freeman qui, sans la publier, a eu une entrevue avec Mead). Mead répond en quelques lignes, en 1969, en maintenant que ces travaux se sont déroulés soit sur d'autres îles de Samoa, soit à une autre époque et ne peuvent donc être comparés à ses propres données. Le débat reste limité à quelques spécialistes de la Polynésie occidentale. La plupart des autres chercheurs en sciences sociales n'en sauront rien.

Pour ce qui est du grand public américain des années trente, l'histoire est plus simple. Il est accroché par tout ce qui parle de "sex »: Freeman a rassemblé un florilège de réactions, avec la minutie qui le caractérise. Ces exemples n'ayant pas été contredits, alors que tant d'articles ont cherché à discréditer la critique de Freeman, on peut estimer qu'ils sont véridiques.

Dès la sortie du livre, les journaux insistent sur le fait que Mead a trouvé un lieu où les problèmes sexuels qui caractérisent l'Occident sont totalement absents ${ }^{33}$. La même année, un autre compte rendu du livre s'intitule "Sex in the South Seas " et appelle à quitter l'Occident pour trouver ces îles merveilleuses, «to find love which is free, easy and satisfying ${ }^{34} »$. «Sex, love» : le mot est dans la presse. Dans des livres aussi on cite Mead pour appeler également à ce retour : «Back to the South Sea Isles », retour au " naturalness and simplicity and sexual joy ${ }^{35}$ ». D'autres parleront de ce livre comme un grand résultat dans «the domain of erotics ${ }^{36} »$ ! Des gens aussi sérieux que Bertrand Russell, parce qu'ils sont des avocats de la libération des mœurs, évoquent l'exemple de Samoa en 1929 et, n'en retenant que l'atmosphère que Mead semble y décrire, en rajoutent : "[Samoan men] when they have to go upon a journey, fully expect their wives to console themselves for their absence ${ }^{37}$ ». C'est un effet significatif de la charmante description par Mead d'« une journée à Samoa». La liste pourrait être allongée à l'infini. Havelock Ellis, "the vénérable seer of the sexually enlightened " selon Freeman, admire cette culture samoane où l'éducation a permis d'éliminer totalement « les névroses, la frigidité et l'impuissance ${ }^{38}$ ».

En outre, dès la publication de son livre et pendant toute sa vie, Mead écrit de très nombreux articles dans des revues touchant à l'éducation, où elle cite constamment l'exemple samoan. Elle alimente ainsi elle-même la tendance à une lecture de son travail réduite aux généralisations. Les titres sont évocateurs: "The Sex Life of the Unmarried Adult in Primitive Society ", dans un livre collectif sur The Sex Life of the Unmarried Adult, publié à Londres en 1935; "Back of Adolescence Lies Early Childhood", dans la revue Childhood Education (1941); "Parents and Children in Samoa ", dans la revue Child Study, et tant d'autres articles du même genre, dans 
Parents' Magazine, Journal of Educational Sociology, etc. ${ }^{39}$ Mais Mead ne se limite pas à l'éducation sexuelle. Tout ce qui est « culturel » la concerne. En 1950, par exemple, elle publie des articles comme «Cultural Contexts of Nutritional Patterns » pour l'American Association for the Advancement of Science, ou "Food and the Family", pour l'Unesco ${ }^{40}$.

Ainsi, aux États-Unis, dans tout le monde anglophone et même ailleurs, ce livre de Mead fut l'étude la plus citée, sans interruption depuis 1928, dans les encyclopédies, les ouvrages de vulgarisation et les débats de toute sorte, dès qu'il s'agissait de parler d'éducation, de sexualité et d'adolescence. Le livre faisait partie du savoir universel, celui qu'on n'a plus à examiner, mais que l'on doit citer.

S'il est bon de réexaminer les savoirs considérés comme acquis, toutes les manières ne se valent pas. Une déconstruction des savoirs en place, comme nous l'avons (à peine) esquissée ici, est profitable, alors que lorsqu'on se contente de lui opposer une "vérité » sociologique telle que D. Freeman la définit en prenant appui sur Popper, cette réduction scientiste de ce que sont une preuve ou une interprétation en sciences sociales aboutit inévitablement à d'autres déformations tout aussi simplificatrices. Le débat « Freeman-Mead » pourrait alors être l'occasion de s'interroger sérieusement sur les moyens dont disposent les sciences sociales pour conduire leur autocritique.

\section{NOTES}

1. Margaret Mead and Samoa. The Making and Unmaking of an Anthropological Myth, Cambridge, Harvard University Press, 1983. L'ouvrage est complété peu après par un article d'une centaine de pages, "Fact and Context in Ethnography: The Samoan Controversy », Canberra Anthropology, 6 (2), 1983, et par deux autres, publiés en 1991, qui reconstituent le parcours de Mead avant, pendant et peu après son séjour à Samoa : « There's Tricks i'th' World. An Historical Analysis of the Samoan Researches of Margaret Mead », Visual Anthropology, 7 (1), 1991, et «On Franz Boas and the Samoan Researches of Margaret Mead », Current Anthropology, 32 (3), 1991.

2. M. Mead, Coming of Age in Samoa, New York, Morrow, 1928 (réédité constamment chez Penguin ; traduction française sous le titre Adolescence à Samoa, in Møurs et sexualité en Océanie, Paris, Plon, 1963, p. 291-503).

3. On ne pouvait rêver meilleur exemple, depuis que l'anthropologie existe, pour illustrer les défauts que J.-C. Passeron stigmatise à juste titre dans Le raisonnement sociologique. L'espace nonpoppérien du raisonnement naturel, Paris, Nathan, 1991 ; il y montre les apories et les dangers de la croyance (le terme s'applique bien à Freeman) en une science sociale qui prétendrait remplacer l'interprétation par des vérités au sens de Karl Popper, c'est-à-dire capables de subir l'épreuve de la « réfutation ». Par ailleurs, nous avons montré, il y a longtemps déjà, à quel point un fait social ne fait sens qu'une fois pris en compte le «niveau» où il se trouve dans la hiérarchie des références (quand une relation sociale surdétermine le fonctionnement d'une autre, la réciproque n'étant pas vraie). En ce sens, le fait d'opposer simplement des contre-exemples à des affirmations annule cette perception hiérarchique et holiste (la place dans le tout, ce dernier étant organisé par ce jeu des surdéterminations) et ne laisse plus en place qu'un seul plan d'analyse, à la manière des «structuralismes » d'outre-Manche qui n'ont comme outillage que 
des «+ » à opposer à des « - ». Sur ces modèles, dont certains travaux de Rodney Needham sont une parfaite illustration, leur critique et le remplacement par un modèle en niveaux, pour toutes les structures dualistes, voir S. Tcherkézoff, Le roi nyamwezi, la droite et la gauche. Révision comparative des classifications dualistes, Cambridge-Paris, Cambridge University Press-Éd. de la Maison des Sciences de l'Homme, 1983, et « Hierarchical Reversais, Ten Years on ", Journal of the Anthropological Society of Oxford, 25, 1994-1995, p. 133-167, 229-253.

4. S. Tcherkézoff, «La question du "genre" à Samoa. De l'illusion dualiste à la hiérarchie des niveaux ", Anthropologie et Sociétés, 16 (2), 1992, p. 91-117; "The illusion of dualism in Samoa: "brothers-and-sisters" are not "men-and-women" ", in T. Del Valle, ed., Gendered Anthropology (Actes de la 1st European Association of Social Anthropologists Conference, août 1990, vol. 4), Londres, Routledge \& Kegan Paul, 1993, p. 54-87; «Qu'est-ce qu'un acte sexuel, au Samoa Occidental? ", dans les Actes du Colloque «Horizon de l'anthropologie et trajets de Maurice Godelier » [Cerisy, 20-27 juin 1996], P. Descola, J. Hamel et P. Lemonnier, eds, Éd. des Colloques de Cerisy, 1998 ; “"Sœur ou épouse, il faut choisir !" L'énigme de l'exogamie villageoise à Samoa : mariage, résidence et asymétrie des sexes dans une société polynésienne ", in F. Héritier et E. Copet-Rougier, eds, Relations de germanité, Paris, Éd. des Archives contemporaines, 1998.

5. S. Tcherkézoff, "Culture, nation, société. Changements secondaires et bouleversements au Samoa occidental. Vers un modèle pour l'étude des dynamiques culturelles ", in S. Tcherkézoff et F. Douaire-Marsaudon, eds, Le Pacifique-sud aujourd'hui. Identités et transformations culturelles, Paris, Éd. du CNRS, 1997, p. 289-351.

6. Sex and Temperament in Three Primitive Societies, publié en 1935, trad. fr., "Trois sociétés primitives de Nouvelle-Guinée (région du Sepik) ", in M. Mead, Mœurs et sexualité..., op. cit., p. V-VI (première page de l'introduction; nous soulignons).

7. M. Mead, Adolescence à Samoa, op. cit., p. 433.

8. Voir la reproduction de cette couverture dans D. Freeman, « There's Tricks i'th' World... », op. cit., p. 118.

9. M. Mead, Adolescence à Samoa, op. cit., p. 306-309.

10. M. Mead, "Americanization in Samoa ", American Mercury, 16, 1929, p. 269, cité à deux reprises par D. Freeman, Margaret Mead and Samoa, op. cit., p. 91, 226.

11. M. Mead, Adolescence à Samoa, op. cit., p. 301-304.

12. Ibid., p. 447 [ $1^{\text {re }}$ cit.], p. 400 [ $2^{\mathrm{e}}$ cit.], p. 431-432 [3 ${ }^{\mathrm{e}}$ cit., nous soulignons].

13. En plus des témoignages que nous avons recueillis sur place, voir l'évocation de ce ressentiment dans l'intervention du professeur samoan Aiono dans le film australien de Frank Heimans, Margaret Mead and Samoa (1987, distribué par Brighton Video, New York).

14. B. Danielsson, Love in the South Seas, New York, Reynal \& Co (éd. originale suédoise, 1954), 1956, p. $55,81,85$.

15. E. S. C. Handy, The Native Culture in the Marquesas, Honolulu, Bernice P. Bishop Museum, 1923, p. 39-40, cité in B. Danielsson, Love in the South Seas, op. cit., p. 93.

16. E. S. C. Handy, Polynesian Religion, Honolulu, Bernice P. Bishop Museum (Bulletin 34), 1927, p. 306-307, cité in B. Danielsson, Love in the South Seas, op. cit., p. 158.

17. R. Linton, "Marquesan Culture", in A. Kardiner, The Individual and his Society, New York, Columbia University Press, 1939, cité par B. Danielsson, Love in the South Seas, op. cit., p. 171.

18. R. H. Lowie dans American Anthropologist, 31, 1929, p. 532. Cette référence et les deux suivantes sont données par D. Freeman, Margaret Mead and Samoa, op. cit., p. 97-98, 226-227, et « On Franz Boas... ", op. cit., p. 325.

19. J. Honigmann, Understanding Culture, New York, Harper \& Row, 1963, p. 273.

20. BBC, « Reith Lectures », 1962.

21. Tous les boasiens font des comptes rendus élogieux du livre de Mead: cf. D. Freeman, Margaret Mead and Samoa, op. cit., chap. 7, et surtout « On Franz Boas... », op. cit., p. 326-327.

22. Cité par D. Freeman, « On Franz Boas... », op. cit., p. 325. 
23. Cité par D. Freeman, ibid., p. 326.

24. Le livre de B. Malinowski est publié l'année suivante: The Sexual Life of Savages in Northwestern Melanesia. An Ethnographic Account of Courtship, Marriage, and Family Life among the Natives of the Trobriand Islands, British New Guinea, Londres, Routledge, 1929 (réédité en 1931, 1932 ; avec une préface d'Annette Weiner, Boston, Beacon Press, 1987).

25. Cité par D. Freeman, « On Franz Boas... », op. cit., p. 327.

26. Seul Sapir, qui trouve le livre "cheap and dull», émet des critiques violentes. La raison pourrait ne pas être que scientifique. Freeman a souligné le fait que Sapir avait eu une liaison avec Mead en 1925 ; elle était alors mariée à un étudiant en théologie, dont elle divorcera à son retour de Samoa. Mead avait repoussé la proposition de mariage faite par Sapir, qui avait tout fait pour l'empêcher de partir pour Samoa : cf. D. Freeman, « On Franz Boas... », op. cit., et "There's Tricks i'th' World », op. cit., p. 107.

27. Cité par D. Freeman, « On Franz Boas... », op. cit, p. 325.

28. M. Mead, Social Organization of Manu'a, Honolulu, Bernice P. Bishop Museum (Bulletin 76), 1930 ; réimpr. Honolulu, P. Bishop Museum Press, 1969.

29. R. Firth, We The Tikopia [1936], Londres, G. Allen and Unwin, 1957, p. XXII.

30. D. Freeman, Margaret Mead and Samoa, op. cit., p. 102.

31. Texte en quatrième de couverture de M. Mead, ed., Cultural Patterns and Technical Change. A Manual prepared by the World Federation for Mental Health, New York, Mentor Books, 1955 (éd. originale par l'Unesco). Le livre porte sur la Grèce, le Nouveau Mexique, le Nigeria, Palau (Micronésie) et fait un peu le point sur les dynamiques culturelles du moment.

32. L. Borghi, « Presentazione al lettore italiano », in M. Mead, L'adolescente in una società primitiva, Florence, Éd. Universitaria, 1964.

33. American Mercury, 15, 1928, p.22. Cette référence et les cinq suivantes sont données par D. Freeman, Margaret Mead and Samoa, op. cit., p. 97-98, 226-227.

34. The Nation, 127, 1928, p. 427.

35. S. D. Schmalhausen, Our Changing Human Nature, New York, The Macaulay Company, 1929, p. 8. 36. F. O'Brien, cité sur la quatrième de couverture de l'édition de 1962 de Coming of Age in Samoa.

37. B. Russell, Marriage and Morals, Londres, G. Allen and Unwin, 1929.

38. H. Ellis, "Introduction" in V.F. Calverton et S. D. Schmalhausen, eds, Sex in Civilization, Londres, 1929. On voit, par tous ces titres des années 1929-1930, que le livre de Mead n'aurait pas pu choisir un meilleur moment pour être publié. Tout un mouvement intellectuel cherchait à poser le principe d'une autre morale de vie. Tous se jetèrent sur le «Samoa » décrit par Mead comme la misère (sexuelle) sur le monde (enchanté).

39. Voir ces références et quelques autres dans D. Freeman, Margaret Mead and Samoa, op. cit., p. 321 , note 7 .

40. Ces titres figurent dans la bibliographie de M. Mead, ed., Cultural Patterns and Technical Change..., op. cit.

\section{RÉSUMÉS}

Le débat le plus intense et le plus volumineux de toute l'histoire de l'anthropologie sociale et culturelle a commencé en 1983, quand les travaux de D. Freeman ont remis en cause l'étude de la culture polynésienne de Samoa effectuée par Margaret Mead en 1928. Le débat s'est centré sur la 
personne de Mead, sur les méthodes des sciences sociales et sur les idées polynésiennes concernant la sexualité. À partir d'une étude sur le terrain de la culture samoane, l'auteur présente les principaux biais de l'analyse de Mead; même si les critiques développées par un professeur australien se portent sur le terrain glissant de l'interprétation en général et sont inconclusives, elles soulignent ce que Mead a totalement manqué dans son étude. L'article s'attache donc à une question centrale: comment s'est constitué le consensus des anthropologues, ainsi que du grand public, autour du travail naif, parfois ridicule, d'une jeune étudiante inexpérimentée ? C'est un moment de l'histoire de l'anthropologie (1925-1950) qui est concerné.

The most intense and extensive debate of the entire history of social and cultural anthropology began in 1983, when the works of D. Freeman questioned Margaret Mead's study of the Polynesian culture of Samoa published in 1928. The debate was centred on the person of Mead, on the methods of the social sciences and on polynesian ideas concerning sexuality. On the basis of a field study of samoan culture, the author presents the principal angles of Mead's analysis; even if the criticisms developed by an Australian professor concern the slippery ground of interpretation in general and are inconclusive, they underline what Mead completely missed in her study. The paper thus deals with a central question : how is the consensus of anthropologists as well as that of the large public constituted around a young inexperienced student's naive, sometimes ridiculous work? A moment of the history of anthropology (1925-1950) is concerned.

\section{AUTEUR}

\section{SERGE TCHERKÉZOFF}

Serge Tcherkézoff (EHESS-Marseille) est anthropologue social et conduit depuis plus de quinze ans des enquêtes en Polynésie occidentale, après avoir travaillé sur l'ethnographie de l'Afrique ancienne. Il a publié un ouvrage sur les formes de pouvoir sacré et les classifications sociales en Afrique de l'Est, de nombreux articles sur l'histoire des modèles anthropologiques et sur la société polynésienne de Samoa ; il a co-dirigé un ouvrage présentant les dynamiques culturelles à l'œuvre dans les pays du Pacifique-sud contemporain. 